\title{
COMPUTER SUPPORTED UBIQUITOUS LEARNING ENVIRONMENT FOR VOCABULARY LEARNING USING RFID TAGS
}

Hiroaki Ogata, Ryo Akamatsu, and Yoneo Yano Dept. of Information Science and Intelligent Systems, Faculty of Engineering, Tokushima University

\begin{abstract}
This paper describes a computer-assisted language learning (CALL) in a ubiquitous computing environment. The environment, called TANGO (Tag Added learNinG Objects) system, detects the objects around the learner using RFID (Radio Frequency Identification) tags, and provides the learner the right information for language learning. This system supports learning in daily life with PDA (personal digital assistant) beyond web based education with desktop computers. In the experiment, the learner played the game of TANGO, and was very interested in this system.
\end{abstract}

Keywords: Computer Supported Ubiquitous Learning, Mobile Learning, Pervasive Learning, Computer Assisted Language Learning, Vocabulary Learning, and RFID tag.

\section{INTRODUCTION}

Ubiquitous computing [1] will help organize and mediate social interactions wherever and whenever these situations might occur [6]. Its evolution has recently been accelerated by improved wireless telecommunications capabilities, open networks, continued increases in computing power, improved battery technology, and the emergence of flexible software architectures. With those technologies, an individual learning environment can be embedded in the real everyday life.

The main characteristics of ubiquitous learning are shown as follows $[3,4]$ :

a) Permanency: Learners never lose their work unless it is purposefully deleted. In addition, all the learning processes are recorded continuously everyday.

b) Accessibility: Learners have access to their documents, data, or videos from anywhere. That information is provided based on their requests. Therefore, the learning involved is self-directed. 
c) Immediacy: Wherever learners are, they can get any information immediately. Thus, learners can solve problems quickly. Otherwise, the learner can record the questions and look for the answer later.

d) Interactivity: Learners can interact with experts, teachers, or peers in the form of synchronous or asynchronous communication. Hence, the experts are more reachable and the knowledge becomes more available.

e) Situating of instructional activities: The learning could be embedded in our daily life. The problems encountered as well as the knowledge required are all presented in their natural and authentic forms. This helps learners notice the features of problem situations that make particular actions relevant.

Moreover, ubiquitous learning can be Computer Supported Collaborative Learning (CSCL) [10] environments that focus on the socio-cognitive process of social knowledge building and sharing.

The challenge in an information-rich world is not only to make information available to people at any time, at any place, and in any form, but specifically to say the right thing at the right time in the right way [5]. A ubiquitous computing environment enables people to learn at any time and any place. But the fundamental issue is how to provide learners with the right information at the right time in the right way. This paper tackles the issues of right time and right place learning (RTRPL) in a ubiquitous computing environment.

This paper proposes TANGO (Tag Added learNinG Objects) System for vocabulary learning. At the beginner's class of language learning, a label that has written the name of the object is stuck on the corresponding object in a room in order to remind learners the word. The idea of this system is that the learner sticks RFID (Radio Frequency Identification) tags on real objects instead of sticky labels, annotate them (e.g., questions and answers), and share them among others. The tags bridge authentic objects and their information in the virtual world. TANGO system detects the objects around the learner using RFID tags, and provides the learner with the right information in that context.

The motivation of this research is based on the first author's experience. As a visiting researcher, he visited the center for lifelong learning and design at the University of Colorado at Boulder in USA for 18 months. Before staying USA, he had learnt English (mainly reading and writing skills using text book) for almost 20 years from junior high school. However, he had a lot of difficulties in the daily life in USA because of the difference between English he had learnt and English in the real world. This means it is necessary not only to learn English with text book, but also to learn it in the real situation. Therefore, in order to solve this problem, this paper proposes computer supported ubiquitous learning for supporting learning in the real 
world. The system facilitates sharing the past experiences, presenting the right question at the right place and the right time by detecting the context of the learning situation, and solving the confront problem immediately by providing the right information via wireless network.

\section{CSUL: COMPUTER SUPPORTED UBIQUITOUS LEARNING}

\subsection{What is CSUL?}

CSUL (Computer Supported Ubiquitous Learning) is defined as a ubiquitous learning environment that is supported by embedded and invisible computers in everyday life. Figure 1 shows the comparison of four learning environments according to [6]. The CAL (computer assisted learning) systems using desktop computers are not embedded in the real world, and are difficult to move. Therefore, those systems hardly support learning at anytime and anywhere.

Compared with desktop computer assisted learning, mobile learning is fundamentally about increasing learners' capability to physically move their own learning environment with them. Mobile learning is implemented with lightweight devices such as PDA (Personal Digital Assistant), cellular mobile phones, and so on. Those mobile devices can connect to Internet with wireless communication technologies, and enable the learning at anytime and anywhere. In this situation, however, computers are not embedded in the learner's surrounding environment, and they cannot seamlessly and flexibly obtain information about the context of his/her learning.

In pervasive learning, computers can obtain information about the context of learning from the learning environment where small devices such as sensors, pads, badges, and so on, are embedded and communicate mutually. Pervasive learning environments can be built either by embedding models of a specific environment into dedicated computers, or by building generic capabilities using computers to inquire, detect, explore, and dynamically build models of the environments. However, this makes availability and usefulness of pervasive learning limited and highly localized.

Finally, ubiquitous learning has integrated high mobility with pervasive learning environments. While the learner is moving with his/her mobile device, the system dynamically supports his/her learning by communicating with embedded computers in the environment. As for the broad definition of ubiquitous learning, both pervasive learning and mobile learning would be in 
the category of ubiquitous learning. RFID tags are often used to realize pervasive computing.

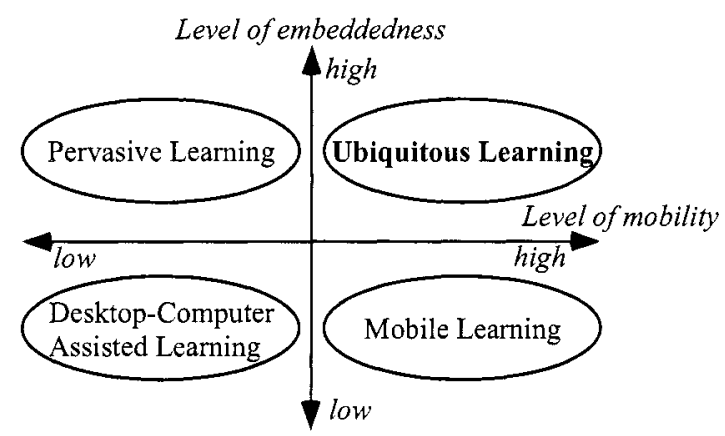

Figure 1. Classification of learning environment. (based on [6]).

\subsection{Learning Theories for CSUL}

CSUL is advocated by pedagogical theories such as on-demand learning, hands-on or minds-on learning, and authentic learning [8,9,10,11]. CSUL system provides learners on-demand information such as advices from teachers or experts at the spot at the precise moment they want to know something. Brown, Collins, and Duguid [2] define authentic learning as coherent, meaningful, and purposeful activities. When the classroom activities are related to the real world, students receive great academic delights. There are four types of learning to ensure authentic learning: action, situated, incidental, and experimental learning [13].

Miller and Gildea [7] worked on vocabulary teaching, and described how children are taught words from dictionary definitions and a few exemplary sentences. They have compared this method with the way vocabulary is normally learned outside school. People generally learn words in the context of ordinary communication. Therefore, we believe that it is very important to support vocabulary learning in everyday life with ubiquitous computing technologies.

\section{TANGO}

We have developed the prototype system called TANGO, which works on a Toshiba Genio-e with Pocket PC 2002, RFID tag reader/writer (OMRON V720S-HMF01), and wireless LAN (IEEE 802.11b). RFID tag reader/writer is attached on a CF (Compact Flash) card slot of PDA as shown in Figure 2. 
The tag unit can read and write data into and from RFID tags within $5 \mathrm{~cm}$ distance, and it works with a wireless LAN at the same time. The TANGO program has been implemented with Embedded Visual Basic 3.0.

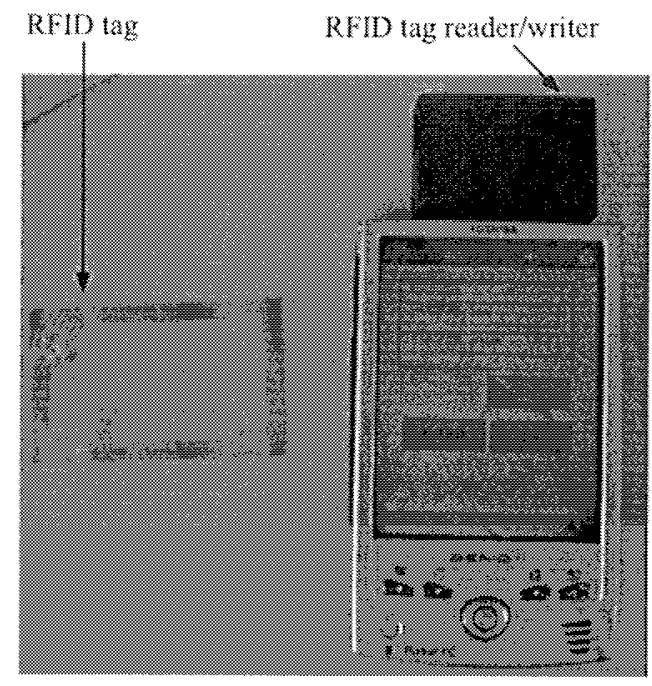

Figure 2. TANGO system.

\subsection{RFID}

Radio frequency identification (RFID) tag is a rewritable IC memory with non-contact communication facility. There are two organizations for promoting this technology.

First, the Auto-ID Center [14] is "designing, building, testing and deploying a global infrastructure - a layer on top of the Internet - that will make it possible for computers to identify any object anywhere in the world instantly. This network will not just provide the means to feed reliable, accurate, real-time information into existing business applications; it will usher into a whole new era of innovation and opportunity."

Second, the Ubiquitous ID Center [15] "aims to establish and spread core technology to automatically recognize "things", and eventually realize a ubiquitous computing environment." This paper presumes objects in the real world have ID tags, and computers can easily identify them.

\subsection{System Configuration}

TANGO has the following modules:

a) Learner model: This module has the learner's profile such as name, age, gender, occupation, interests, etc, and the comprehensive level of each 
word or each expression about an object. Before using TANGO, the learner does the examination, and enters the comprehensive level. In addition to this explicit method, TANGO detects learner's comprehension during the system use.

b) Environmental model: This module has the data of objects, rooms and buildings, and the link between objects and expressions in the learning materials database.

c) Educational model: This module manages words and expressions as learning materials. The teacher enters the fundamental expressions for each object. Then, both learners and teacher can add or modify them during system use.

d) Communication tool: This tool provides the users with a BBS (bulletin board system) and a chat tool, and stores their logs into a database.

e) Tag reader/writer: This module reads the ID from a RFID tag attached to an object. Referring to the ID in the object database, the system obtains the name of the object.

f) User interface: This module provides learner questions and answers.
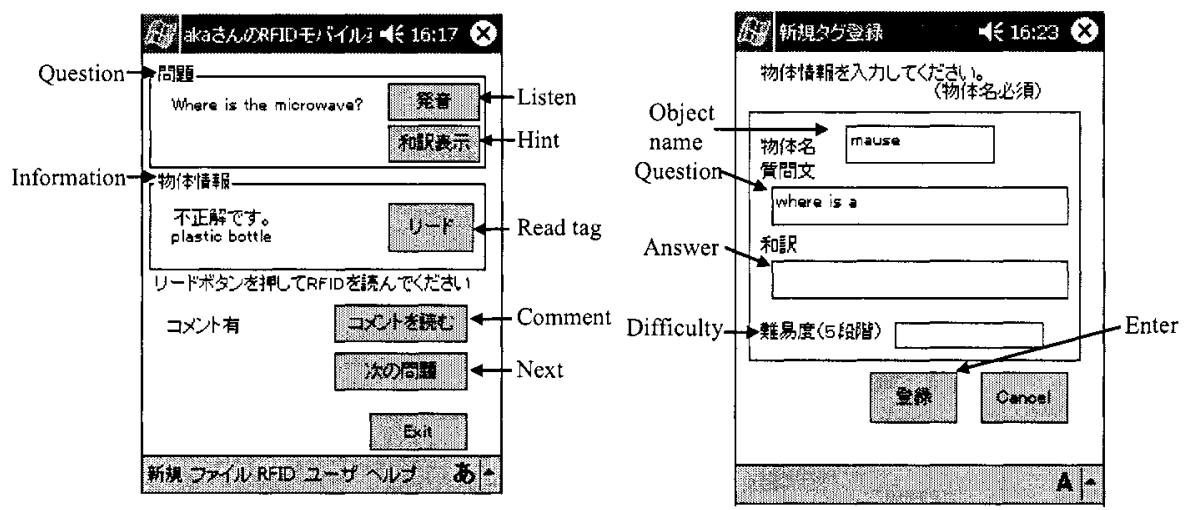

Figure 3. Main window and new-tag window of TANGO.

\subsection{User Interface}

The interface of the learning environment TANGO is shown in Figure 3 and 4. In figure 3, TANGO system asks the learner a question with voice. The adequate question is selected by scanning the RFID tags around the learner and using the learner model. If the learner cannot understand the question, the learner can listen again by clicking the button. Furthermore, if the learner cannot understand the question, the system shows the text of the question as a hit by clicking "hint button." By clicking "read button", the system begins to read a tag. If the learner scans the correct tag, the answer is 
right. Then, the learner can move to the next question by pushing "next button." If the object has a comment, the comment button is active. By clicking "comment button", the comment appears as shown in figure 5. The learner can also add comment by clicking "add comment" button (see in figure 6). The learner has RFID tags, can attach them on objects, and create a new question about the new object as shown in figure 4. In this way, learners can collaboratively learn language by sharing comments and tags.
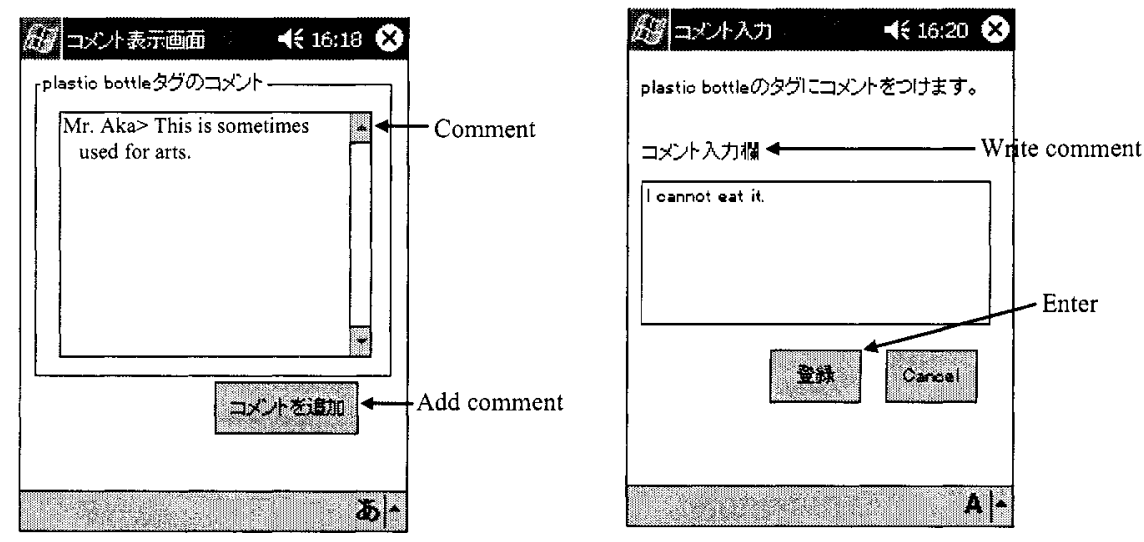

Figure 4. Comment window of TANGO.

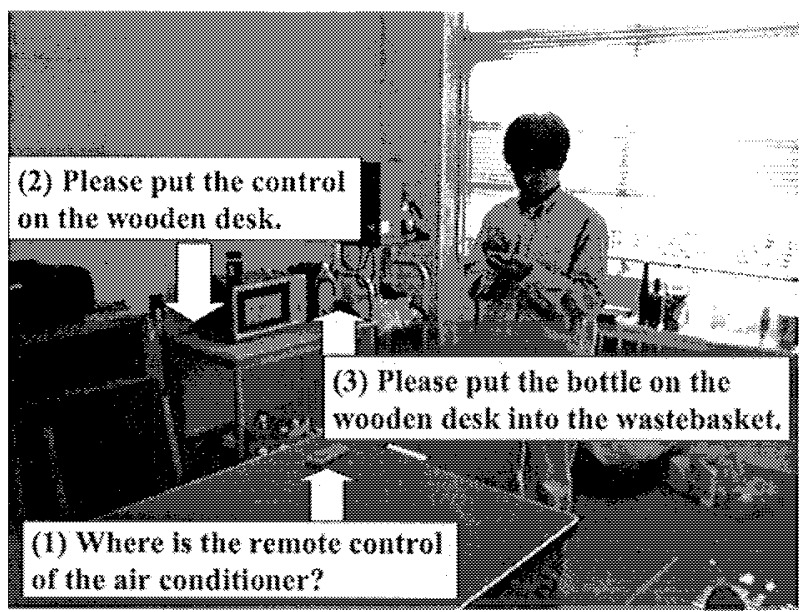

Figure 5. Usage example of TANGO. 
Figure 5 shows an example of a room where RFID tags were attached to some objects for TANGO. For example, when the learner enters a meeting room, the system asks him/her the question "Where the remote control of the air conditioner is?". The learner can hear the question again if s/he wants. Moreover, the learner can see the text and hints. If the learner scans the tag labeled on the remote control, the answer is correct. Then the system will ask to put it on the wooden desk. This way, the interaction between the learner and the system goes on.

Table 1: The results of questionnaires.

\begin{tabular}{cccc}
\hline No. & Questionnaire & Ave. & S.D. \\
Q1 & $\begin{array}{l}\text { Were the questions provided by this system } \\
\text { difficult? }\end{array}$ & 3.33 & 0.52 \\
Q2 & $\quad$ Do you think this system easy to use? & 3.33 & 1.03 \\
Q3 & $\begin{array}{l}\text { Do you think this system useful for language } \\
\text { learning? }\end{array}$ & 4.17 & 0.41 \\
Q4 & Is the response of this system is adequate to use? & 3.67 & 0.52 \\
Q5 & Do you think this system very interesting? & 4.33 & 0.52 \\
Q6 & Do you want to keep using this system? & 3.83 & 0.75 \\
\hline
\end{tabular}

\section{EXPERIMENTATION}

We arranged six high school students to evaluate TANGO with a questionnaire. The average of the users' age was 16 . The users had no PDA, and 50 percent of them had their own computers. We explained them how to use the PDA, before they used TANGO. Each user looked for objects in the room according to the questions read aloud by the PDA. For example, if the question was where the fabric chair was in the room, and. the user scans the RFID tag of the chair, the user gets the point. Otherwise, the user loses the point. Users competed against each other for getting the points. After the experiment, they gave a score between one and five to each of nine questions, with one being the lowest, and five being the highest. The average score was 3.78. Table 1 shows the results of the questionnaire. According to question (1), some of the users felt that the questions were difficult. Question (2) shows that TANGO system is not so easy to use. That is because this experiment was the first time for all the users to use a PDA. However, the user interface should be improved for the novice user who does not have her/his own PDA.

In terms of language learning, question (3) shows that TANGO was quite useful for it. One of the learners commented that with this system, it was easy to understand the terms by their correspondence with authentic objects. 
From the results of question (4), we should make the response of the system a little faster. The questions (5) and (6) show the users were very interested in this system, and that they would like to keep using it. Some learners commented that they had a feeling of achievement.

\section{CONCLUSIONS}

This paper described a computer-assisted language learning (CALL) in an ubiquitous computing environment. TANGO system detects the objects around the learner using RFID tags, and provides the learner with the educational information. In the experiment, the learner played the game of TANGO, and was very interested in this system.

\section{ACKNOWLEDGEMENT}

This work was partly supported by the Grant-in-Aid for Scientific Research No.15700516 from the Ministry of Education, Science, Sports and Culture in Japan.

\section{REFERENCES}

[1] Abowd, G.D., and Mynatt, E.D.: Charting Past, Present, and Future Research in Ubiquitous Computing, ACM Transaction on Computer-Human Interaction, Vol.7, No.1, pp.29-58, 2000.

[2] Brown, J. S., Collins, A., and Duguid, P.: Situated Cognition and the Culture of Learning. Educational Researcher, ( Jan.-Feb.), pp.32-42, 1989.

[3] Chen, Y.S., Kao, T.C., Sheu, J.P., and Chiang, C.Y.: A Mobile Scaffolding-Aid-Based Bird -Watching Learning System, Proceedings of IEEE International Workshop on Wireless and Mobile Technologies in Education (WMTE'02), pp.15-22, IEEE Computer Society Press, 2002.

[4] Curtis, M., Luchini, K., Bobrowsky, W., Quintana, C., and Soloway, E.: Handheld Use in K-12: A Descriptive Account, Proceedings of IEEE International Workshop on Wireless and Mobile Technologies in Education (WMTE'02), pp.23-30, IEEE Computer Society Press, 2002.

[5] Fischer, G.: User Modeling in Human-Computer Interaction, Journal of User Modeling and User-Adapted Interaction (UMUAI), Vol. 11, No.1/2, pp.65-86, 2001.

[6] Lyytinen, K. and Yoo, Y.: Issues and Challenges in Ubiquitous Computing, Communications of ACM, Vol.45, No.12, pp.63-65, 2002.

[7] Miller, G. A., and Gildea, P.M.: How children learn words. Scientific American, No.257, pp.94-99, 1987.

[8] Ogata, H., and Yano, Y.: How Ubiquitous Computing can Support Language Learning, Proc. of KEST 2003, pp. 1-6, 2003. 
[9] Ogata, H., and Yano, Y.: Supporting Knowledge Awareness for a Ubiquitous CSCL, Proc. of E-Learn 2003, pp.2362-2369, 2003.

[10] Ogata, H., and Yano, Y: Knowledge Awareness Map for Computer-Supported Ubiquitous Language-Learning, Proc. of IEEE WMTE2004, Taiwan, 2004. (in press)

[11] Ogata, H., and Yano, Y: Context-Aware Support for Computer-Supported Ubiquitous Learning, Proc. of IEEE WMTE2004, Taiwan, 2004. (in press)

[12] O'Malley, C.: Computer supported collaborative learning, NATO ASI Series, F: Computer \& Systems Sciences, Vol.128, 1994.

[13] Hwang. K.S.: Authentic Tasks in Second Language Learning, http://tiger.coe.missouri.edu/ vlib/Sang's. htm

[14] Auto ID center: http://www.autoidcenter.org

[15] Ubiquitous ID center: http://www.uidcenter.org 